ORIGINAL ARTICLE

\title{
Modification of plasma glycosaminoglycans in long distance runners
}

\author{
M Contini, S Pacini, L Ibba-Manneschi, V Boddi, M Ruggiero, G Liguri, M Gulisano, C Catini
}

Br J Sports Med 2004;38:134-137. doi: 10.1136/bjsm.2002.001388

See end of article for authors' affiliations

.....................

Correspondence to: Dr Pacini, Department of Anatomy, Histology and Forensic Medicine,

University of Firenze, Viale Morgagni 85-50134,

Florence 50134, Italy;

stefania.pacini@unifi.it

Accepted

18 February 2003
Background: It is well documented that exercise reduces the risk of thromboembolic disease, possibly by increasing the plasma concentration of anticoagulant-antithrombotic compounds.

Objectives: As plasma glycosaminoglycans (GAGs) play a role in the anticoagulant-antithrombotic potential of plasma, to examine the concentration and profile of these compounds in well trained, long distance runners and sedentary subjects.

Methods: Plasma GAGs were measured in 10 male, long distance runners and 10 sedentary counterparts before and after ergometric tests. GAGs were extracted, purified, and identified by electrophoretic and enzymatic methods, and measured as hexosamine.

Results: Plasma GAGs found in sedentary subjects were slow migrating heparan sulphates I and II, keratan sulphate I, and chondroitin 4-6-sulphate. Those found in trained athletes were slow migrating heparan sulphate I, chondroitin 4-6-sulphate (or keratan sulphate I), and fast migrating heparan sulphate. Total plasma concentrations of GAGs were higher in athletes than in sedentary subjects at rest. In sedentary subjects, plasma GAGs did not change after cycle ergometric exercise at $80 \%$ of their anaerobic threshold. However, the appearance of a novel band of heparan sulphate migrating faster than fast migrating heparan sulphate was observed in athletes after exercise.

Conclusions: Exercise changes the amount and profile of plasma GAGs; these changes may play a role in protecting subjects who practise aerobic sports against developing cardiovascular disease.
${ }^{\mathrm{t}}$ $\mathrm{t}$ is a common perception that physical activity protects against the development of cardiovascular disease. Several epidemiological studies confirm this idea, and regular exercise appears to reduce the risk of thromboembolism and vascular disease. ${ }^{1}$ A possible link between exercise and reduced risk for cardiovascular disease was identified in "beneficial" variations occurring in several variables of the coagulation system after prolonged exercise. Thus, it has been shown that fibrinolytic activity and all the circulating anticoagulants increased after near maximum exercise; only relatively prolonged effort, however, could trigger a mechanism beneficial to the cardiovascular system. ${ }^{2}$ The mechanism by which regular exercise affects coagulation variables remains incompletely understood.

We hypothesised that well trained, long distance runners may have different amounts and profile of plasma glycosaminoglycans (GAGs), which may lead to favourable variations in haemostatic variables. Consistent with this hypothesis, Roos et al showed that serum concentrations of certain GAGs, hylaluronan and keratan sulphate, were higher in runners than healthy controls or soccer players, and further increased after exercise. Other studies have shown that the plasma concentration of GAGs depends on the physical training of the subjects examined, being significantly higher in athletes than in sedentary people. $^{4}$

It is worth noting that plasma GAGs play a role in maintaining the antithrombotic potential of normal human plasma, ${ }^{56}$ and we recently characterised circulating anticoagulant GAGs in normal human plasma. ${ }^{7}$ To determine whether exercise could actually modify the amount and profile of plasma GAGs in healthy subjects, we measured plasma GAGs in sedentary subjects and well trained, long distance runners before and after exercise.

\section{METHODS}

\section{Subjects}

Two groups of subjects were studied. The first was composed of 10 healthy men, aged 20-30, whose lifestyle could be defined as sedentary: none regularly engaged in strenuous exercise or hard physical work. The second was composed of 10 healthy, well trained, male, long distance runners, aged 18-34, with average training distance $90-130 \mathrm{~km}$ a week. Table 1 shows the physical characteristics of all the subjects.

Before being enrolled in the study, all subjects had a detailed medical examination, and only those showing no evidence of disease were included. They were asked not to take any drugs for at least three weeks before the day when blood was sampled or the day of the ergometry test, and not to change their usual dietary habits and level of physical activity. The dietary habits of the subjects did not differ significantly from those of the adult Italian population. All subjects gave written informed consent to the study according to the institutional review committee guidelines on human subjects.

\section{Blood samples}

For the evaluation of plasma GAGs, resting and fasting venous blood samples were taken from each sedentary subject and athlete, after they had avoided major physical activity for the preceding 24 hours. Each blood sample $(9 \mathrm{ml})$, obtained by plastic syringe from the antecubital vein, was anticoagulated with $1 \mathrm{ml}$ buffered sodium citrate $(0.11 \mathrm{~mol} / \mathrm{l})$. Subjects were seated during blood withdrawal. Blood samples were taken at the beginning (phase A) and

Abbreviation: GAG, glycosaminoglycan 
Table 1 Physical characteristics and ventilatory and metabolic variables of sedentary subjects $(n=10)$ and athletes $(n=10)$

\begin{tabular}{|c|c|c|c|c|c|c|}
\hline & \multicolumn{3}{|c|}{ Sedentary subjects } & \multicolumn{3}{|c|}{ Athletes } \\
\hline & Mean & Median & SD & Mean & Median & SD \\
\hline Age (years) & 24.0 & 23.5 & 3.1 & 25.9 & 26.5 & 5.4 \\
\hline Weight (kg) & 63.0 & 64.0 & 10.1 & 61.6 & 60.0 & 6.2 \\
\hline Height $(\mathrm{cm})$ & 171.3 & 170.0 & 10.5 & 172.8 & 172.0 & 7.8 \\
\hline $\mathrm{BSA}\left(\mathrm{m}^{2}\right)$ & 1.73 & 1.75 & 0.19 & 1.73 & 1.70 & 0.15 \\
\hline $\mathrm{BMI}\left(\mathrm{kg} / \mathrm{m}^{2}\right)$ & 21.3 & 21.5 & 1.5 & 20.6 & 20.7 & 0.8 \\
\hline $\mathrm{VO}_{2} \mathrm{MAX}(\mathrm{ml} / \mathrm{min})$ & 2397 & 2254 & 331 & 3622 & 3646 & 396 \\
\hline$V_{E}(I / \mathrm{min})$ & 74.1 & 69.5 & 12.1 & 117.8 & 120.5 & 14.2 \\
\hline AT $\left(\% \mathrm{VO}_{2} \mathrm{MAX}\right)$ & 61.5 & 59.5 & 6.4 & 81.4 & 82.4 & 8.7 \\
\hline
\end{tabular}

BSA, Body surface area, calculated as $0.007184 \times$ weight $^{0.425} \times$ height ${ }^{0.725}$; BMI, body mass index calculated as the Quetelet index as weight/height ${ }^{2} ; \mathrm{VO}_{2} \mathrm{MAX}$, maximum oxygen consumption; $\mathrm{VE}$, expiratory ventilation; $\mathrm{AT}\left(\% \mathrm{VO}_{2} \mathrm{MAX}\right)$, percentage of $\mathrm{VO}_{2} \mathrm{MAX}$ corresponding to anaerobic threshold.

end (phase B) of the cycle ergometry test and in the recovery stage, when $\mathrm{VCO}_{2}$ had returned to baseline (phase $\mathrm{C}$ ).

\section{Isolation and purification of GAGs from plasma}

Platelet-poor plasma was obtained by centrifugation at $2500 \mathrm{~g}$ for 10 minutes at $20^{\circ} \mathrm{C}$. The samples were lyophilised, weighed (dry weight), and subjected to lipid extraction with diethyl ether for 24 hours at $4^{\circ} \mathrm{C} .{ }^{8}$ After rehydration of the samples, proteins were denatured and digested with Pronase E (Protease type XXV; Sigma Chemical Co, St Louis, Missouri, USA) for six hours at $57^{\circ} \mathrm{C}$; the digestion procedure was repeated five times. After treatment with $0.5 \mathrm{M} \mathrm{NaOH}$ for 24 hours at $4^{\circ} \mathrm{C}$, the residual proteins were precipitated with cold $\left(4^{\circ} \mathrm{C}\right)$ trichloroacetic acid and removed by centrifugation at $1500 \mathrm{~g}$ for 20 minutes. Then, each sample was dialysed against running water for 48 hours, against deionised water, and finally against distilled water for 24 hours at $4^{\circ} \mathrm{C}$. After further lyophilisation and lipid extraction with diethyl ether, the samples were solubilised, $\mathrm{NaCl}(0.03 \mathrm{M})$ was added, and GAGs were precipitated using cetylpyridinium chloride (Sigma), at $4^{\circ} \mathrm{C}$ for $24-48$ hours. GAG-cetylpyridinium chloride complexes were removed by centrifugation at $1500 \mathrm{~g}$ for 20 minutes and washed three times with $80 \%$ ethanol saturated with sodium acetate and three times with $80 \%$ ethanol.

\section{Quantification and identification of plasma GAGs}

GAGs were measured in terms of hexosamine content (mg/ $\mathrm{ml})^{9}$ using $N$-acetyl-D-glucosamine (Sigma) as standard. They were identified by the enzymatic/electrophoretic micromethod. The electrophoresis was carried out using $0.1 \mathrm{M}$ barium acetate solution and cellulose polyacetate strips (Gelphore; Gelman Instruments, Wrightsville, Pennsylvania, USA), at $5 \mathrm{~V} / \mathrm{cm}$ for 180 minutes. ${ }^{10}$ Electrophoretic patterns were visualised by staining with Alcian Blue $8 \mathrm{GX}^{11}$ (Sigma) and recorded using a densitometer (ERV Proteo 125). Electrophoretic bands were identified by selective enzymatic digestion with specific hyaluronidase (Sigma), chondroitinase ABC (Sigma) and AC (Sigma), heparitinase (Miles Laboratories, Elkhart, Indiana, USA), and keratanase (Sigma), and by degradation with nitrous acid. Appropriate standards (keratan sulphate, heparan sulphate, and chondroitin 4-6-sulphate) were from Sigma. Human GAGs isolated from umbilical cord, spleen, and bone were also used as standards. ${ }^{12} 13$

\section{Exercise protocol of submaximal ergometric test}

After verifying normal respiratory activity, all the subjects performed two ergometric tests in two different periods. The first was an incremental ergometric test on an electromagnetically braked cycle ergometer (STS 3 Cardioline). After muscle adaptation and warm up, the initial work load of $50 \mathrm{~W}$ was increased by $30 \mathrm{~W}$ every two minutes, until exhaustion. During the test, heart rate was measured by continuous electrocardiogram recording. Standard techniques of open circuit spirometry were used to collect ventilatory and metabolic data. Respired gases were sampled through a low resistance breathing mask to measure oxygen uptake $\left(\mathrm{VO}_{2}\right)$, carbon dioxide output $\left(\mathrm{V}_{\mathrm{CO}_{2}}\right)$, expiratory ventilation $(\mathrm{VE})$, tidal volume, and respiratory exchange ratio. These variables were continuously analysed and computed breath by breath during each exercise stage with a gas analyser (MMC4400TC; Sensor Medics, Yorba Linda, California, USA). This test allowed calculation, by the ventilatory method, of the anaerobic threshold and the maximum oxygen consumption $\left(\mathrm{VO}_{2} \mathrm{MAX}\right)$ for each subject (table 1). One week after the first test, all the subjects underwent a second ergometry test. This was performed for 12 minutes at a constant load equivalent to $80 \%$ of the anaerobic threshold assessed during the first test.

\section{Statistical analysis}

Plasma GAG concentrations were analysed by split-plot analysis of variance.

\section{RESULTS}

Table 2 gives total plasma GAG concentrations, measured in terms of hexosamine, in sedentary subjects and trained runners at rest.

The electrophoretic pattern of GAGs from sedentary subjects showed four bands, starting from the cathode (fig lA). The first and second band were identified as slow migrating heparan sulphate I and II respectively, as they comigrated with the appropriate standard and disappeared after treatment with nitrous acid (fig 1B). The third band disappeared after treatment with keratanase and was therefore identified as keratan sulphate I (fig IC). The fourth band was identified as chondroitin 4-6-sulphate, as it co-migrated with the appropriate standard and disappeared after treatment with chondroitinase AC (fig 1D). The electrophoretic patterns were uniform, with few quantitative variations. As measured by densitometry, the first band, corresponding to the slow migrating heparan sulphate I, comprised, on average, $10 \%$ of the total GAGs $\left(3.3 \times 10^{-5} \mathrm{mg} / \mathrm{ml}\right)$; the second band, identified as slow migrating heparan sulphate II, comprised $5 \%\left(1.6 \times 10^{-5} \mathrm{mg} / \mathrm{ml}\right)$; the third band, corresponding to keratan sulphate I, comprised $27 \%$ $\left(9.1 \times 10^{-5} \mathrm{mg} / \mathrm{ml}\right)$; and the fourth band, corresponding to chondroitin 4-6-sulphate, comprised $57 \%\left(18.9 \times 10^{-5} \mathrm{mg} / \mathrm{ml}\right)$.

Plasma concentrations of GAGs in the trained athletes at rest were significantly higher $(p<0.00005)$ than in the sedentary subjects. The electrophoretic pattern showed three bands which, starting from the cathode, were identified as

Table 2 Plasma glycosaminoglycans, measured in terms of hexosamine $(\mathrm{mg} / \mathrm{ml})$, in sedentary subjects and athletes, before $(A)$ and after $(B)$ the ergometric test, and when $\mathrm{VCO}_{2}$ had returned to baseline (C)

\begin{tabular}{llllllll}
\hline & \multicolumn{3}{l}{ Sedentary subjects } & \multicolumn{3}{l}{ Athletes } \\
\cline { 2 - 3 } & Mean & Median & SD & & Mean & Median & SD \\
\hline A & 3.33 & 3.30 & 0.40 & & 4.92 & 4.70 & 0.81 \\
B & 2.99 & 3.00 & 0.31 & 4.55 & 4.40 & 0.74 \\
C & 3.04 & 3.05 & 0.31 & 4.61 & 4.40 & 0.74 \\
\hline
\end{tabular}

Data are expressed as value $\times 10^{-4}$

The means for the sedentary subjects are significantly lower than those for the athletes $(p<0.00005)$. 


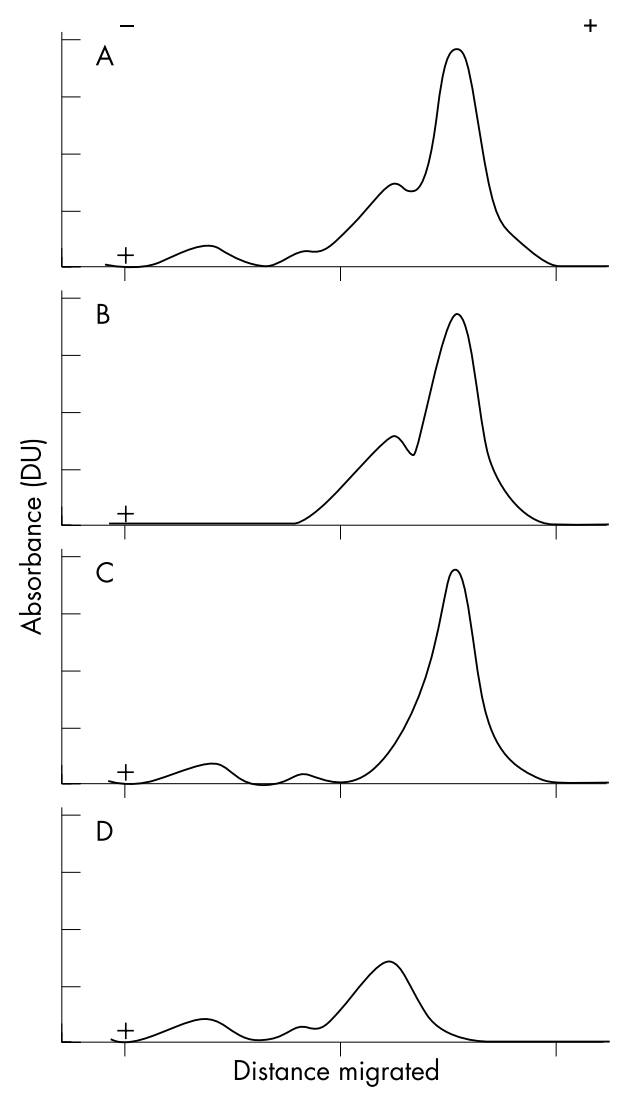

Figure 1 Electrophoretic analysis of plasma glycosaminoglycans in healthy sedentary male subjects on a cellulose polyacetate strip with $0.1 \mathrm{M}$ barium acetate solution at $5 \mathrm{~V} / \mathrm{cm}$ for 180 minutes. (A) Starting from the cathode, slow migrating heparan sulphates I and II, keratan sulphate I, and chondroitin 4-6-sulphate; (B) after treatment with nitrous acid the first two bands disappeared confirming that they corresponded to heparan sulphate; (C) after treatment with keratanase the third band disappeared, thus it was identified as keratan sulphate I; (D) after treatment with chondroitinase $A C$ the fourth band disappeared, thus it was identified as chondroitin 4-6-sulphate. DU, Arbitrary densitometric units.

slow migrating heparan sulphate I, chondroitin 4-6-sulphate (or keratan sulphate I), and fast migrating heparan sulphate (fig 2). The second band was identified as chondroitin 4-6sulphate in six subjects, and as keratan sulphate I in four subjects. On average, the first band, slowly migrating heparan sulphate I, comprised $10 \%$ of total GAGs $\left(4.9 \times 10^{-5} \mathrm{mg} / \mathrm{ml}\right)$; the second band, chondroitin 4-6-sulphate (or keratan sulphate I), comprised $45 \%\left(22.5 \times 10^{-5} \mathrm{mg} / \mathrm{ml}\right)$; the third band, fast migrating heparan sulphate, comprised $45 \%$ $\left(21.9 \times 10^{-5} \mathrm{mg} / \mathrm{ml}\right)$.

After the second ergometry test, the total plasma GAG concentration did not change significantly in either sedentary subjects or athletes (table 2). However, athletes showed significant variations in the electrophoretic pattern, as shown by the appearance of a novel band in phases $\mathrm{B}$ and $\mathrm{C}$, in all the athletes examined; this band migrated faster than fast migrating heparan sulphate (fig 3). As measured by densitometry, this band comprised on average $9 \%$ of total plasma GAGs $\left(4.6 \times 10^{-5} \mathrm{mg} / \mathrm{ml}\right)$. Two of these bands were found in six subjects, whereas in the others only one preband was present. This preband behaved similarly to heparan sulphate when submitted to heparitinase and nitrous acid degradation (fig 3B-D).

\section{DISCUSSION}

"Beneficial" changes in the clotting state of the blood have previously been identified in athletes in aerobic sports such as
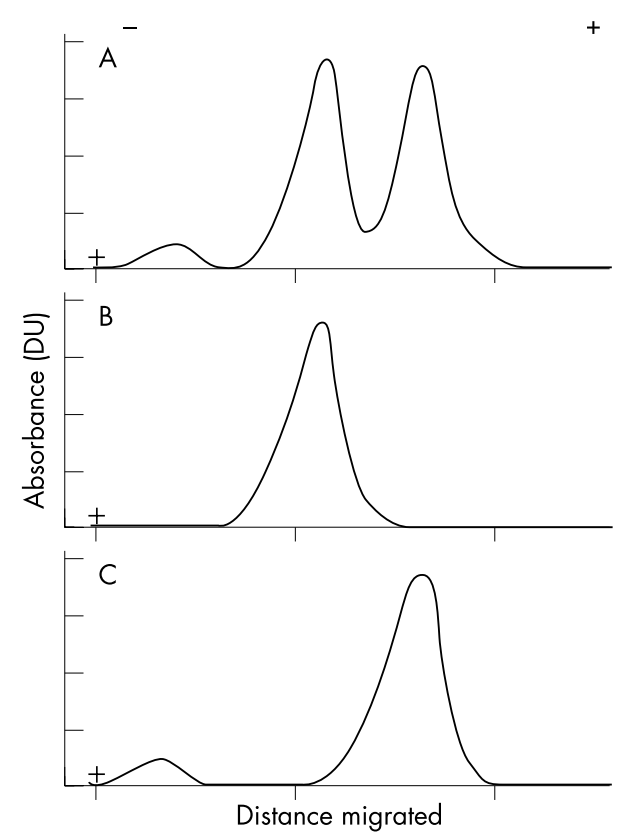

Figure 2 Electrophoretic analysis of plasma glycosaminoglycans of long distance runners on a cellulose polyacetate strip with $0.1 \mathrm{M}$ barium acetate solution at $5 \mathrm{~V} / \mathrm{cm}$ for 180 minutes. (A) starting from the cathode, the slow migrating heparan sulphate; (B) after treatment with nitrous acid, the first and the third band disappeared; (C) after treatment with keratanase the second band disappeared. DU, Arbitrary densitometric units.

marathon running and rowing. ${ }^{2}$ These changes may be similar to those that protect regularly exercising middle aged and older men against cardiovascular disease. ${ }^{114}$ We show here that long distance runners have different amounts and profile of plasma GAGs from sedentary healthy subjects. Firstly, the athletes had higher concentrations of total GAGs than the sedentary controls. Secondly, in the profile of electrophoretic migration, a band corresponding to fast migrating heparan sulphate was observed in athletes, but not in sedentary subjects. Moreover, after the cycle ergometry test, prebands migrated faster than fast migrating heparan sulphate bands in athletes, but not in sedentary subjects. As highly sulphated GAGs would precipitate in barium acetate, we assume that these fast migrating heparan sulphate species were low molecular mass compounds.

It has previously been shown that, low molecular mass heparan sulphates are powerful endogenous antithrombotic agents. Their effects are exerted primarily by inhibiting Factors Xa and IIa, acting as activators of antithrombin III. ${ }^{6}{ }^{15}$ Interestingly, the profile of plasma GAGs in the athletes is similar to that of a novel antithrombotic drug, danaparoid sodium (Orgaran; Organon, Oss, the Netherlands); ${ }^{16}$ this is a low molecular mass heparinoid consisting of heparan sulphate, dermatan sulphate, and chondroitin sulphate, recently approved for prophylaxis of postoperative deep vein thrombosis in patients having hip replacement surgery. ${ }^{17}$

The mechanism by which regular exercise increases the concentration of heparan sulphate and other GAGs remains

\section{Take home message}

Exercise changes the amount and profile of plasma glycosaminoglycans; these changes may play a role in protecting aerobic athletes against the development of cardiovascular disease. 


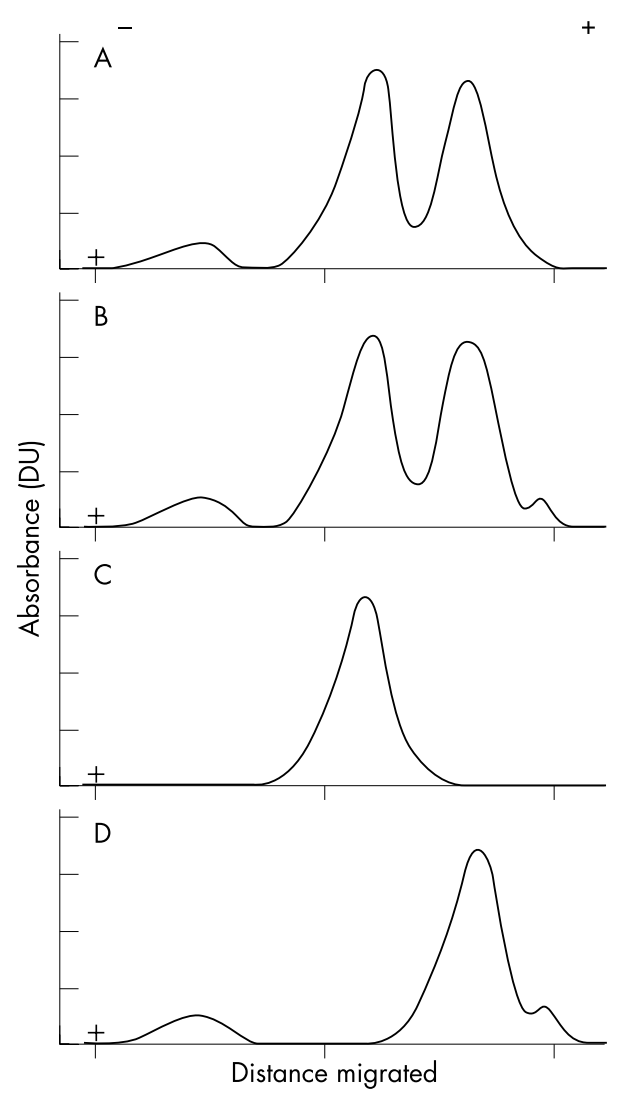

Figure 3 Electrophoretic modification of plasma glycosaminoglycans after ergometric exercise in long distance runners. (A) Pattern before ergometry test; (B) modification after ergometry test (a new band on the right of the band corresponding to fast migrating heparan sulphate appeared); (C) this preband disappeared after treatment with nitrous acid, thus it was identified as heparan sulphate; (D) the sample was treated with keratanase before electrophoresis. DU, Arbitrary densitometric units.

to be elucidated. Previous observations have suggested that a higher turnover rate of body cartilage matrix may account for the increase in hylaluronan and keratan sulphate in serum after exercise. ${ }^{3}$ Other authors have suggested that intramuscular connective tissue is the main site of origin of plasma GAGs in athletes. ${ }^{4}$ However, the presence of low molecular mass compounds in athletes supports the hypothesis that a processive assembly of specific sequences in heparan sulphate may occur at a higher level in athletes than in sedentary subjects. ${ }^{18}$ Regular physical activity, in particular that typical of aerobic sports, may induce higher levels of expression of the genes involved in heparan sulphate biosynthesis leading to the generation of specific saccharide sequences.

In conclusion, we have shown that long distance runners have greater amounts and different composition of plasma GAGs compared with sedentary subjects; we postulate that these changes may be responsible for the variations in blood coagulation variables observed in athletes, which may reduce their risk of cardiovascular disease.

\section{ACKNOWLEDGEMENTS}

This study was supported by grants from the University of Firenze. SP received a grant for young researchers. We are indebted to Benedetta Peruzzi for helpful suggestions and technical help.

\section{Authors' affiliations}

M Contini, S Pacini, L Ibba-Manneschi, M Gulisano, C Catini,

Department of Anatomy, Histology and Forensic Medicine, University of Firenze, Florence, Italy

V Boddi, M Ruggiero, Department of Experimental Pathology and Oncology, University of Firenze

G Liguri, Department of Biochemical Sciences, University of Firenze

\section{REFERENCES}

1 Abbot RD, Rodriguez BL, Burchfield CM, et al. Physical activity in older middle-aged men and reduced risk of stroke: the Honolulu Heart Program. Am J Epidemiol 1994; 139:881-93.

2 Cerneca F, Crocetti G, Gombacci A, at al. Variations in hemostatic parameters after near-maximum exercise and specific tests in athletes. J Sports Med Phys Fitness 1999;39:31-6.

3 Roos H, Dahlberg L, Hoerrner LA, et al. Markers of cartilage matrix metabolism in human joint fluid and serum: the effect of exercise. Osteoarthritis Cartilage 1995:3:7-14.

4 Muraca U, Vinci R, Ferlazzo AM, et al. Factors affecting glycosaminoglycan concentration in normal human plasma. Ital J Biochem 1992;41:159-69.

5 Kolset SO, Solmivirta M. Cell surface heparan sulfate proteoglycans and lipoprotein metabolism. Cell Mol Life Sci 1999;56:857-70.

6 Messa G, La Placa G, Puccetti L, et al. Effectiveness and tolerability of heparan sulfate in the treatment of superficial thrombophlebitis. Controlled clinical study vs sulodexide. Minerva Cardioangiol 1997;45:147-53.

7 Ruggiero $M$, Melli M, Parma B, et al. Isolation of endogenous anticoagulant $\mathrm{N}$-sulphated glycosaminoglycans in human plasma from healthy subjects. Pathophysiol Haemost Thromb 2002;32:44-9.

8 Vannucchi S, Fibbi G, Pasquali F, et al. Adhesion-dependent heparin production by platelets. Nature 1982;296:352-3.

9 Ryan RO, Wessler AN, Price HM, et al. Insect lipid transfer particle catalyzes bidirectional vectorial transfer of diacylglycerol from lipophorin to human low density lipoprotein. J Biol Chem 1990;265:10551-5.

10 Lee KB, Kim JS, Yoo YC, et al. Isolation and characterisation of proteoglycans derived from human placenta and its biological activities. Arch Pharm Res 2000;23:182-6.

11 Zacharius RM. Quantitative determination of hexosamine in glycoprotein by ion-exchange chromatography. J Chromatogr 1976;125:421-7.

12 Catini C, Gheri G. The glycosaminoglycans in the human umbilical cord. Arch Ital Anat Embriol 1983;88:359-73.

13 Catini C, Gheri G, Miliani A. Glycosaminoglycans in the spleen of normal humans and in the spleen of subjects with chronic myeloid leukemia. Nouv Rev Fr Hematol 1984;26:309-15.

14 Diehm C, Morl H, Schettler G. Modification of blood coagulation and fibrinolysis through physical activity. Klin Wochenschr 1984:62:299-302

15 Giorgetti PL, Marenghi MC, Bianciardi P. Heparan sulfate in the therapy of postphlebitic syndrome. Evaluation of the efficacy and tolerability as compared to mesoglycan. Minerva Cardioangiol 1997:45:279-84.

16 Von Lupke U, Marx A, Tessmann R, et al. Danaparoid (Orgaran) as an anticoagulant for mechanical autotrasfusion with Cell Saver (Hemonetics). Anaesthesist 2001;50:26-31.

17 Acostamadiedo JM, lyer UG, Owen K. Dapanaroid sodium. Expert Opin Pharmacother 2000;1:803-14.

18 Zhang L, Yoshida K, Liu J, et al. Anticoagulant heparan sulfate precursor structures in F9 embryonal carcinoma cells. J Biol Chem 1999;274:5681-91.

\section{COMMENTARY}

This paper compares the plasma glycosaminoglycans of sedentary subjects and trained athletes. The two groups were characterised by clinical examination and ventilatory and metabolic variables. The glycosaminoglycans were identified by their electrophoretic mobility and digestion with specific enzymes or deamination with nitrous acid. The results obtained showed a clear difference in the plasma glycosaminoglycans of sedentary subjects and trained athletes, which may correlate with the observation that exercise reduces the risk of thrombosis. It is an interesting publication for those working in the field of the physiology of exercise and prevention of cardiovascular disease.

P A S Mourao Universidade Federal do Rio de Janeiro, Departamento de Bioquimica, Caixa Postal 68041, Rio de Janeiro RJ 21941-500, Brazil; pmourao@hucff.ufri.br 